\title{
Multi-Secondary Transformer: A Modeling Technique for Simulation - II
}

\author{
A. Patel *, N. P. Singh*, L. N. Gupta *, B. Raval *, K. Oza *, A. Thakar *, D. Parmar *, \\ H. Dhola *, R. Dave *, V. Gupta *, S. Gajjar **, P. J. Patel *,U. K. Baruah*
}

\begin{abstract}
Power Transformers with more than one secondary winding are not uncommon in industrial applications. But new classes of applications where very large number of independent secondaries are used are becoming popular in controlled converters for medium and high voltage applications. Cascade H-bridge medium voltage drives and Pulse Step Modulation (PSM) based high voltage power supplies are such applications. Regulated high voltage power supplies (Fig. 1) with 35$100 \mathrm{kV}, 5-10 \mathrm{MW}$ output range with very fast dynamics ( $\mu \mathrm{S}$ order) uses such transformers. Such power supplies are widely used in fusion research. Here series connection of isolated voltage sources with conventional switching semiconductor devices is achieved by large number of separate transformers or by single unit of multi-secondary transformer. Naturally, a transformer having numbers of secondary windings ( 40) on single core is the preferred solution due to space and cost considerations.

For design and simulation analysis of such a power supply, the model of a multi-secondary transformer poses special problem to any circuit analysis software as many simulation softwares provide transformer models with limited number (3-6) of secondary windings. Multi-Secondary transformer models with 3 different schemes are available. A comparison of test results from a practical Multi-secondary transformer with a simulation model using magnetic component is found to describe the behavior closer to observed test results. Earlier models assumed magnetising inductance in a linear loss less core model although in actual it is saturable core made-up of CRGO steel laminations. This article discusses a more detailed representation of flux coupled magnetic model with saturable core properties to simulate actual transformers very close to its observed parameters in test and actual usage.
\end{abstract}

\section{Introduction}

In places where numbers of isolated voltage sources are required, multi-secondary transformers provide compact technical solution for medium power applications. Electrical isolation levels can be achieved by configuring the windings in various dielectric mediums. For the reasons, multi-secondary transformers are widely used in medium voltage industrial drives [1] and modular high voltage power supplies [2]-[6].

To analyze the behavior of multi-secondary transformers an example of modular high voltage power supply is presented. Generalized scheme is shown in Fig. 1 where a typical multi-secondary transformer with 40 numbers of secondary windings is deployed, while its output is shown also in Fig. 2 [4].

As these power supplies are controlled by feedback \& feed forward loop methods, circuit simulation study is natural recourse to predict its behavior during design. PSIM (by Powersim Inc.) is widely used software for circuit

* NBI Group, Institute for Plasma Research Gandhinagar, India. (ampatel@ipr.res.in)

** Power Supply Group, Institute for Plasma Research, ITER-India. Received 11 December 2013; Accepted 12 January 2014

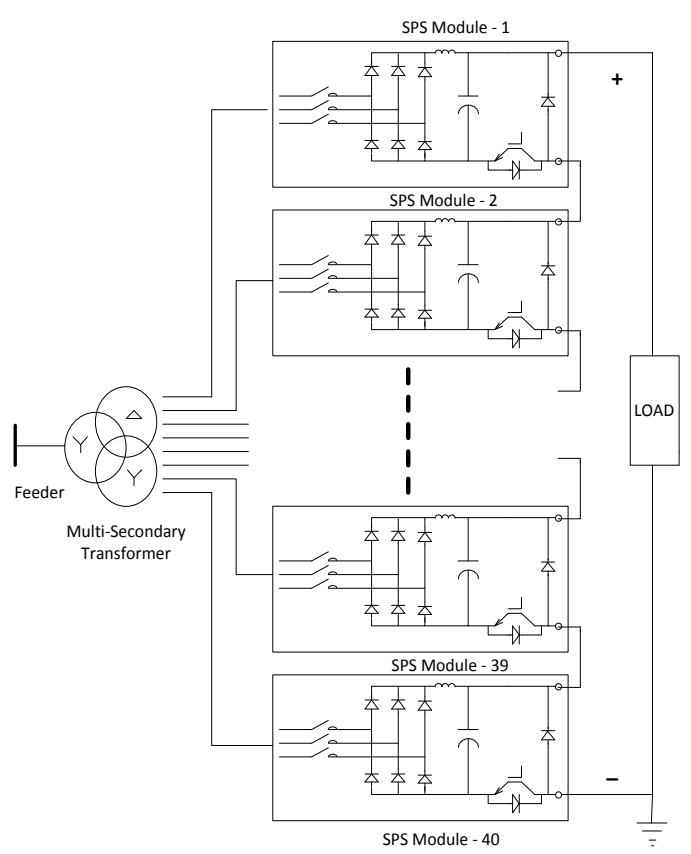

Fig. 1. Pulse Step Modulated HVPS

simulation, where simulation of switching components is faster and reasonably accurate [10]. However multisecondary transformers beyond six secondaries cannot be modeled with the available elements in PSIM's library. To 
overcome this problem, different modeling approaches are discussed, one of them being magnetic model with linear loss-less core in PSIM [7]. In practice transformers are manufactured with core that is made up of CRGO steel laminations working close to the limits of its linear behavior with acceptable losses. This paper presents comparative study of all approaches [7] and mainly discusses modeling of multi-secondary transformer with saturable core.

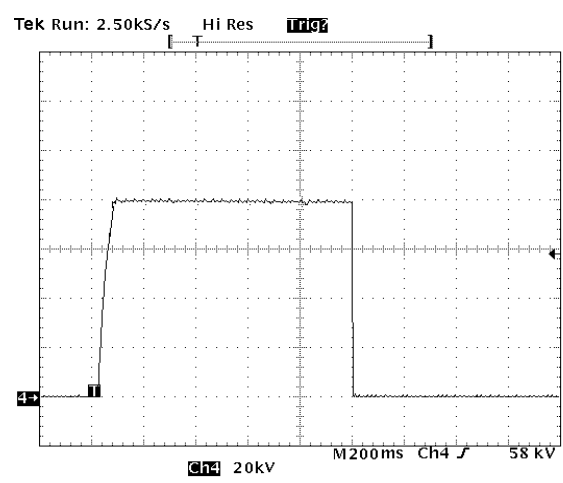

Fig. 2. PSM Based HVPS Application

\section{Multi-Secondary Transformer \& Modeling Approaches}

Measurements were made on a 3-phase, $1600 \mathrm{kVA}, 50 \mathrm{~Hz}$, $11 / 1.1 \mathrm{kV}, 40$ secondary cast resin transformer [8], to be used in a High Voltage Power Supply rated 100kV, $25 \mathrm{~A}$ as shown in Fig. 3. Tests were conducted as per standard norms [9]. During testing, it was found that overall impedance $(\% \mathrm{Z})$ measured is $8 \%$ while measured impedance with individually loaded secondary winding is in $2 \%-3.5 \%$ range. For impedance measurement of individual loaded secondary winding, rated secondary current is passed through by that winding by injecting equivalent primary current in $\mathrm{HV}$ winding. The $2 \%$ value is observed for windings at the middle of a limb and gradually increases up to $3.5 \%$ for top and bottom secondary windings as shown in Fig.4. The actual value of impedances offered by a winding is important to estimate short circuit protection scheme for each secondary winding and ripple voltage on DC link, in particular under no or light load conditions. Circuit model of such a transformer is possible in several ways.

The simplest way to simulate the multi-secondary transformer is to use as many parallel connected individual transformer as secondary windings [7]. But, in this model, each transformer behaves independently, unlike practical multi-secondary transformer where ampere-turn loadings are closely coupled among secondary windings. Another approach by inserting intermediate transformer [7] provides better matching of impedances under individual loaded secondary to all secondaries loaded.

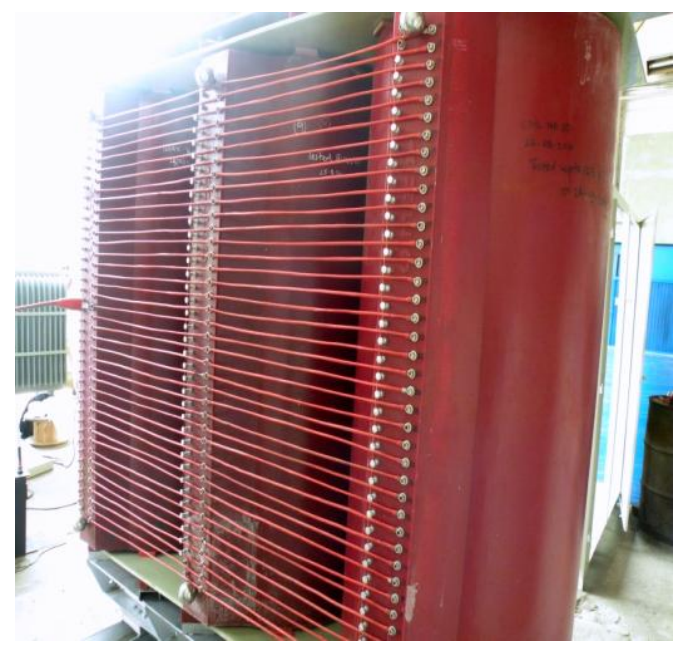

Fig. 3. 1600 kVA, 40 Secondary Transformer

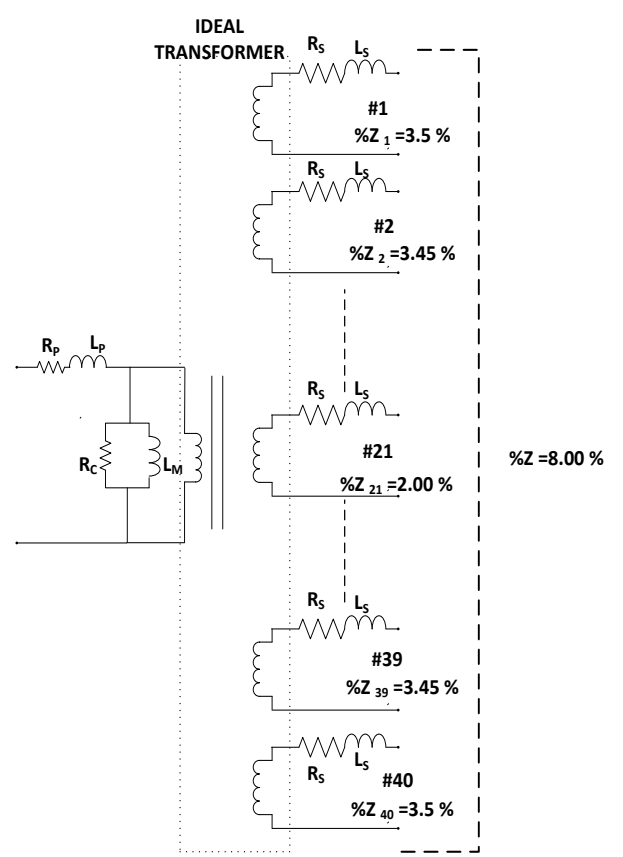

Fig. 4. Secondary Impedance Observation in $1600 \mathrm{kVA}$ Transformer

An improved model of a multi-secondary transformer uses a magnetic element [7] that gives nearly same numerical effect of loading of two windings as a practical multi-secondary transformer. In that approach, for a transformer where overall impedance $(\% \mathrm{Z})$ is observed to be around $8 \%$ while measured impedance with individually loaded secondary winding is $2 \%$.This model too doesn't describe a real transformer, the variation of impedance of windings from the middle to the ends of the limbs are not 
reflected in the result. In practice, this variation in $\% \mathrm{Z}$ for these secondary windings varies from $2-3.5 \%$ (Fig. 4). The model using magnetic elements as in [7] has been revised with use of saturable core in-place of linear loss less core models. In order to get impedance variation due to location of winding on the limb, a gradient in leakage flux is introduced in place of constant leakage flux all throughout.

\section{Model using Revised Magnetic Elements}

A revised model of multi-secondary transformer is simulated in PSIM software. PSIM model library have magnetic elements and saturable core models [10]. B-H curve tool of PSIM software is used to match B-H curve of actual transformer core made up of CRGO steel laminations. The transformer model in PSIM \& its partial sub circuits are shown in Fig. 5. Fig. 5 (right) shows a sub circuit for single phase only while in actual similar 3 networks exists for 3 phase transformer.

A saturable core in PSIM component library require certain parameters are calculated from available transformer dimensions while coefficients are obtained from curve fitting tool with known boundary conditions.

PSIM having the following parameter set for defining a saturable core.

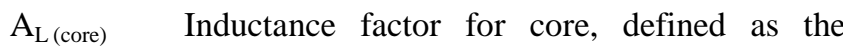
inductance per turn squared, in $\mathrm{H}$,

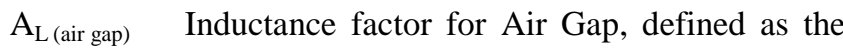
inductance per turn squared, in $\mathrm{H}$,

$\mathrm{A}_{\mathrm{C}} \quad$ Core cross sectional area,

$1_{\mathrm{g}} \quad$ Air Gap Length,

$1 \quad$ Core Length,

$\mathrm{R}_{\mathrm{LC}} \quad$ Core resistance for loss estimate,

C Coefficient $\phi$ sat, in Weber,

Where $\phi_{\text {sat }}=\mathrm{B}_{\text {sat }} * \mathrm{~A}_{\mathrm{C}}$

$\mathrm{K}_{1} \quad$ Coefficient $\mathrm{K}_{1}$ for the core $\mathrm{B}-\mathrm{H}$ curve,

$\mathrm{K}_{\text {exp1 }} \quad$ Coefficient for the core B-H curve,

$\mathrm{K}_{2} \quad$ Coefficient for the core B-H curve,

$\mathrm{K}_{\exp 2} \quad$ Coefficient for the core B-H curve,

Coefficient $\mathrm{K}_{1}, \mathrm{~K}_{\exp 1}, \mathrm{~K}_{2}, \mathrm{~K}_{\exp 2}$ are used to fit B-H curve of actual used steel laminations. All coefficients are for piecewise linearization of B-H curve for CRGO steel. The actual transformer made using M120-27S5 (IEC 60404-8-7 referred nomenclature) grade steel laminations and was designed at Flux density (B) of $\sim 1.5 \mathrm{~T}$. In B-H curve tool box of PSIM Software, Curve fitted by inserting actual dimension data and adjusting various curve fitting coefficients. Fig. 6 shows CRGO material B-H curve obtained by PSIM tool, used for this analysis.

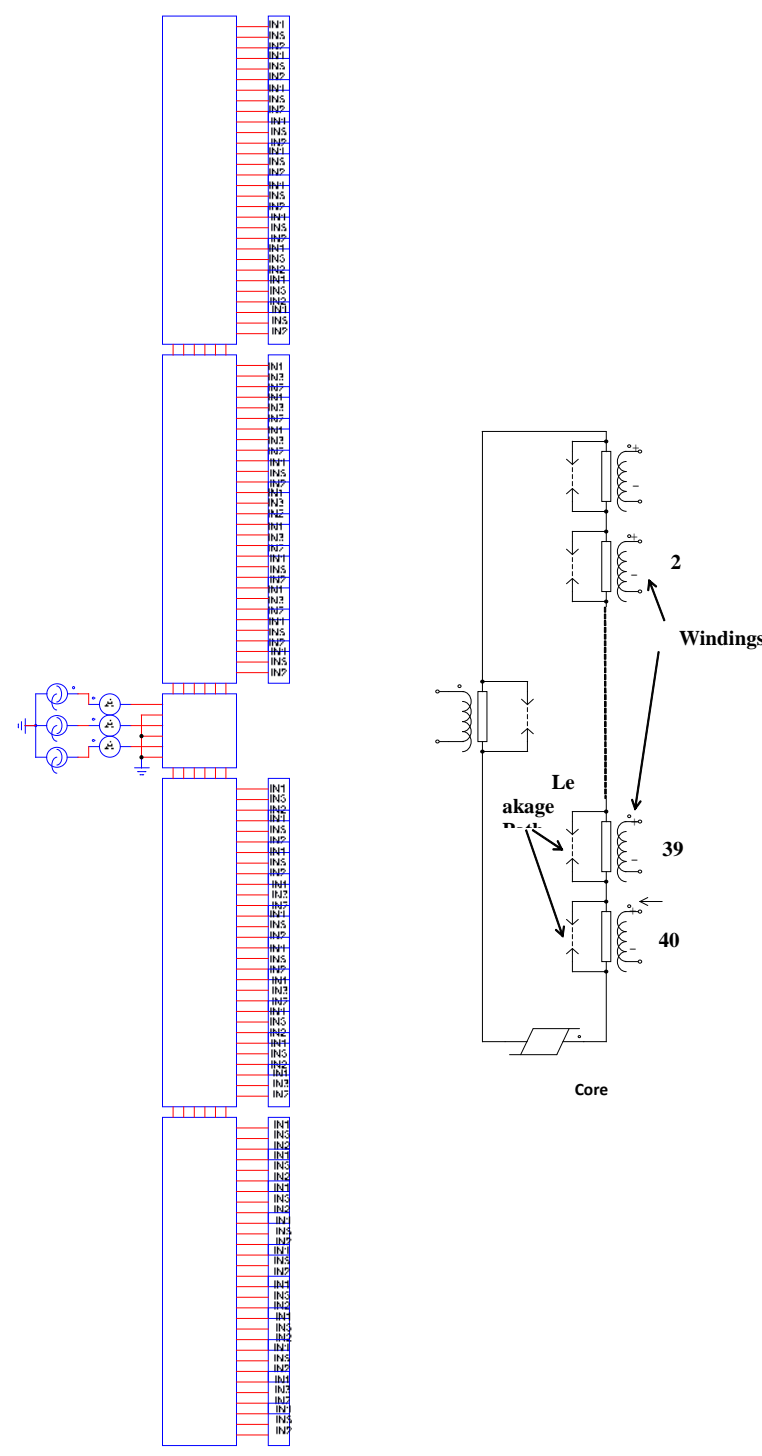

Fig. 5. Multi-Secondary Transformer Magnetic Model

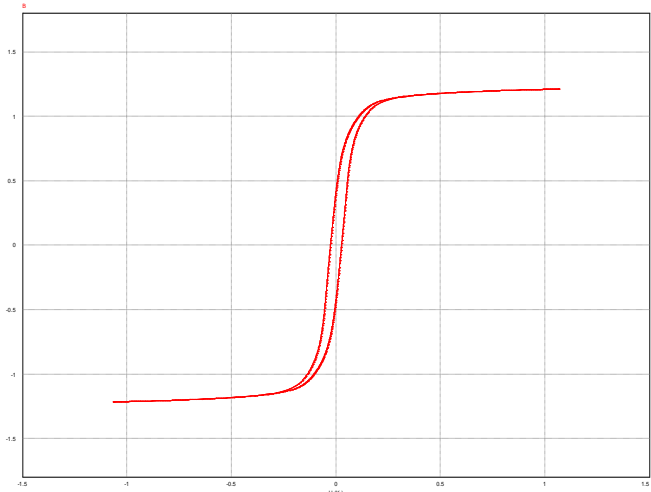

Fig.6. Modeled B-H Curve 
Apart from saturable core parameters, required parameters for this model in PSIM software are,

$\mathrm{R}_{\mathrm{EUQ}} \quad$ Equivalent winding resistance derived from test report,

$\mathrm{L}_{\mathrm{EQU}} \quad$ Equivalent leakage inductance derived from test report,

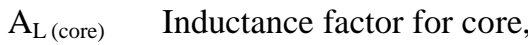

$\mathrm{N}_{\mathrm{P}} \quad$ Number of turns for primary winding,

$\mathrm{R}_{\mathrm{P}} \quad$ Primary winding resistance,

$\mathrm{N}_{\mathrm{S}} \quad$ Number of turns for secondary winding,

$\mathrm{R}_{\mathrm{S}} \quad$ Secondary winding resistance,

$\mathrm{A}_{\mathrm{LP} \text { (Leakage) }}$ Inductance factor for primary winding,

$\mathrm{R}_{\mathrm{LP}} \quad$ Winding resistance due to leakage flux,

$A_{L S}$ (Leakage) Inductance factor for secondary winding,

$\mathrm{R}_{\mathrm{LS}} \quad$ Secondary winding resistance due to leakage flux,

Above parameters can be derived from design data and test data of the available $1600 \mathrm{kVA}, 11 / 1.1 \mathrm{kV}$ transformer. Values of winding resistance $\left(R_{P} \& R_{S}\right)$ and leakage inductance $\left(\mathrm{L}_{\mathrm{P}} \& \mathrm{~L}_{\mathrm{S}}\right)$ are derived from short circuit load test results. $\mathrm{N}_{P}$ and $\mathrm{N}_{S}$ are design parameters. Inductance factor $\left(\mathrm{A}_{\mathrm{LP} \text { (leakage) }}\right)$ is the ratio of leakage inductance to square of number of turns.

$$
\begin{aligned}
& \mathrm{R}_{\mathrm{P}}=\mathrm{R}_{\mathrm{S}}=0.5 * \mathrm{R}_{\mathrm{EQU}} \\
& \mathrm{L}_{\mathrm{P}}=\mathrm{L}_{\mathrm{S}}=0.5 * \mathrm{~L}_{\mathrm{EQU}} \\
& \mathrm{A}_{\mathrm{LP} \text { (Leakage) }}=\mathrm{L}_{\mathrm{P}} /\left(\mathrm{N}_{\mathrm{P}}\right)^{2} \\
& \mathrm{~A}_{\mathrm{LS} \text { (Leakage) }}=\mathrm{L}_{\mathrm{S}} /\left(\mathrm{N}_{\mathrm{S}}\right)^{2}
\end{aligned}
$$

After formation of complete model, it is verified and cross checked with initial necessary simulated tests like Voltage ratio ,Overall impedance (by short circuit test) and no-load losses.

\section{Observation}

Main two observations are done after implementing saturable core model in PSIM,

\section{Individual Secondary Winding Impedance}

Fig.7 shows comparison of individual secondary winding impedance for actual transformer and simulation model. These values are derived from amount of leakage flux linkages to secondary windings in different proportions with respect to position of winding on limb.

\section{No-Load Current}

In transformer, no load current contains odd harmonics because of nonlinear behavior of core material. After implementation of saturable core in model, no-load current is observed and compared with actual transformer results. Table 1 shows FFT spectrum of no-load current for actual transformer \& simulation model while Fig. 8 shows simulation waveform of no-load current.

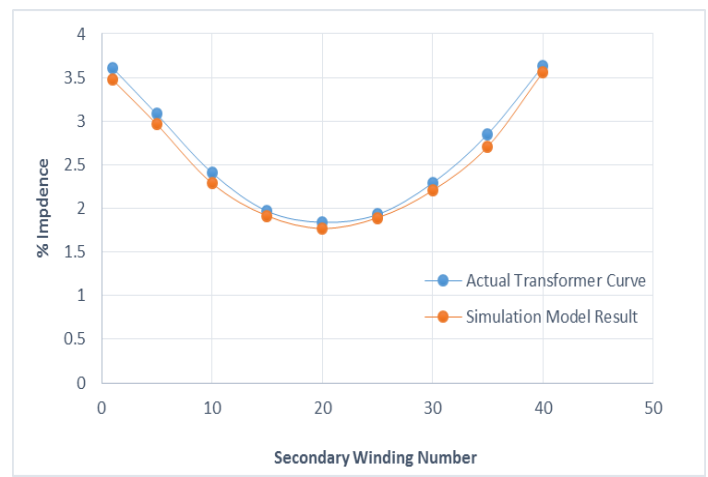

Fig.7. Individual Winding Impedance Comparisons

Table 1. FFT Spectrum of No-Load Current

\begin{tabular}{|c|c|c|}
\hline \multirow{2}{*}{$\begin{array}{c}\text { Harmonic } \\
\text { Order }(\mathrm{n})\end{array}$} & Actual Transformer & Simulation Model \\
\cline { 2 - 3 } & Ratio $\left(\mathrm{I}_{\mathrm{n}} / \mathrm{I}_{1}\right)$ & Ratio $\left(\mathrm{I}_{\mathrm{n}} / \mathrm{I}_{1}\right)$ \\
\hline 1 & 100.00 & 100.00 \\
\hline 3 & 8.10 & 4.60 \\
\hline 5 & 22.00 & 14.20 \\
\hline 7 & 8.50 & 5.10 \\
\hline 9 & 0.30 & 0.80 \\
\hline 11 & 0.75 & 0.57 \\
\hline 13 & 0.26 & 0.10 \\
\hline 15 & 0.15 & 0.08 \\
\hline
\end{tabular}

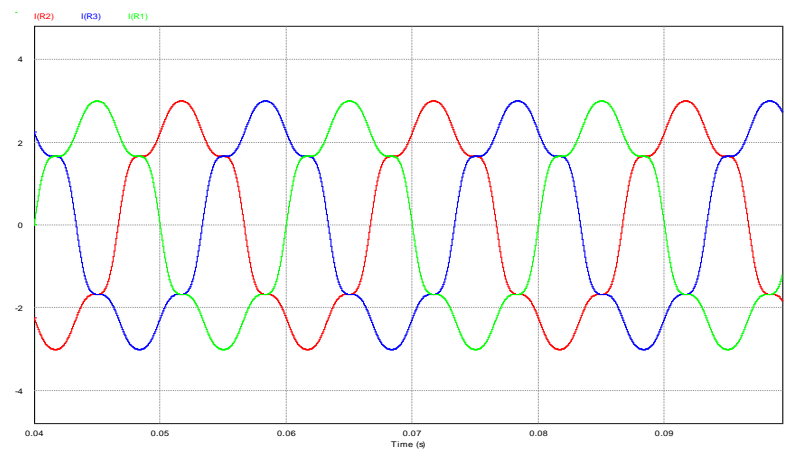

Fig. 8. No-Load Current Waveform

\section{Conclusion}

Even though the conclusion may review the main results or contributions of the paper, do not duplicate the abstract o $r$ the introduction. For the conclusion, you might elaborate on the importance of the work or suggest the potential appli cations and extensions. 


\section{Acknowledgements}

Authors are thankful to M/s. Ames Impex Electricals Pvt. L td., Ahmedabad, India for manufacturing and providing test results of $1600 \mathrm{kVA}, 11 \mathrm{kV} /(1.1 \mathrm{kVx} 40)$ multi secondary cast resin transformer.

\section{References}

[1] Young-Min Parky, Han-Seong Ryu, Hyun-Won Lee, MyungGil Jung and Se-Hyun Lee, "Design of a cascaded H-bridge multilevel inverter based on power electronics building blocks and control for high performance", Journal of Power Electronics, vol. 10, no. 3, May 2010.

[2] Alex J.Schminke W, "Fast switching modular high voltage DC/AC power supplies for RF amplifiers and other applications," Proceeding in 16th IEEE/NPSS Symposium on Fusion Engineering, SOFE 1995. vol. 2, pp. 936-939.

[3] Alex J.Schminke W, "A high voltage power supply for negative ion NBI based on PSM technology," Proceeding in 17th IEEE/NPSS Symposium on Fusion Engineering, 1997. vol. 2, pp. 1063.

[4] P.J. Patel, N.P. Singh, V. Tripathi, L.N. Gupta, and U.K. Baruah, "A regulated high voltage power supply for accelerator driven system", International Topical Meeting on Nuclear Research Applications and Utilization of Accelerators, May 2009, Vienna.

[5] Xu Weidong, Xuan Weimin,Yao Lieying, Wang Yingqiao, "Development of $8 \mathrm{MW}$ power supply based on pulse step modulation technique for auxiliary heating system on HL2A", Plasma Science and Technology, vol.14, no.3, Mar. 2012

[6] D. Fasela, F.Albajar, T.Bonicelli, A.Perez, L.Rinaldi, U.Siravo, L.Sita, G.Taddia, "5MW CW supply system for the ITER gyrotrons Test Facility," Fusion Engineering and Design, 2011.

[7] A.Patel, et al, "Multisecondary transformer: a modeling technique for simulation," IEEE Transaction on Plasma Science, vol. 40, no. 7, pp. 1957-1961, July 2012.

[8] Tripathi V, Singh N.P., Gupta L.N., Oza K., Patel P., Baruah U.K., "Development of cast resin multisecondary 1600kVA transformer for regulated high voltage power supply - a prototype," 23rd National Symposium on Plasma Science \& Technology (PLASMA-2008).

[9] IEC 60076, Power Transformers.

[10] PSIM user manual, version 8.0 (by Powersim Inc.).
A. Patel NBI Group, Institute for Plasma Research Gandhinagar, India. 